\title{
Erratum: Electron-phonon interactions from first principles [Rev. Mod. Phys. 89, 15003 (2017)]
}

\author{
Feliciano Giustino \\ Q (published 11 January 2019) \\ DOI: 10.1103/RevModPhys.91.019901
}

In Fig. 1(d) the electron-phonon coupling function $g$ should appear on the left of the vertex $\Gamma$. The correct figure is

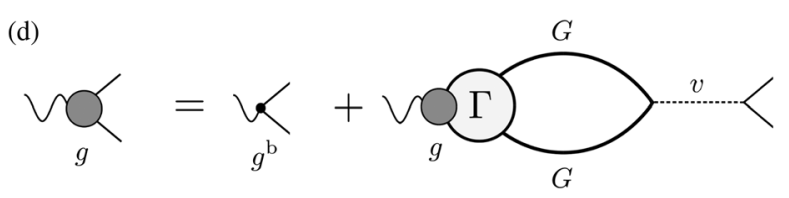

In order to be consistent with the definition of the electron-phonon matrix element in Eq. (38), the factor $N_{p}^{-1 / 2}$ should be removed from Eqs. (2), (57), (58), (61), and (180). This factor is already included in the definition of the electron-phonon Hamiltonian in Eq. (37).

The current density defined on p. 46 misses the volume $\Omega$. The correct expression is

$$
\mathbf{J}=-\frac{2 e}{\Omega} \sum_{n} \int \frac{d \mathbf{k}}{\Omega_{\mathrm{BZ}}} \mathbf{v}_{n \mathbf{k}} f_{n \mathbf{k}} .
$$

In Eq. (202) the sum should also run over the band index $m$, and the terms within the square brackets should be divided by $f_{n \mathbf{k}}^{0}\left(1-f_{n \mathbf{k}}^{0}\right)$ and $f_{m \mathbf{k}+\mathbf{q}}^{0}\left(1-f_{m \mathbf{k}+\mathbf{q}}^{0}\right)$, respectively. The correct equation is

$$
\frac{\partial f_{n \mathbf{k}}^{0}}{\partial \varepsilon_{n \mathbf{k}}} \mathbf{v}_{n \mathbf{k}} \cdot(-e) \mathbf{E}=-\sum_{m \nu} \int \frac{d \mathbf{q}}{\Omega_{\mathrm{BZ}}} \Gamma_{m n \nu}(\mathbf{k}, \mathbf{q})\left[\frac{f_{n \mathbf{k}}-f_{n \mathbf{k}}^{0}}{f_{n \mathbf{k}}^{0}\left(1-f_{n \mathbf{k}}^{0}\right)}-\frac{f_{m \mathbf{k}+\mathbf{q}}-f_{m \mathbf{k}+\mathbf{q}}^{0}}{f_{m \mathbf{k}+\mathbf{q}}^{0}\left(1-f_{m \mathbf{k}+\mathbf{q}}^{0}\right)}\right] .
$$

I am indebted to Yan Wang, Weng Hong Sio, Carla Verdi, and Wenbin Li for bringing these issues to my attention. 